\title{
Bernstein equiconvergence and Fejér type theorems for general rational Fourier series
}

\author{
P. Van gucht and A. Bultheel ${ }^{1,2}$ \\ Department of Computer Science, K.U.Leuven, \\ Celestijnenlaan 200A, B-3001 Heverlee, Belgium
}

\begin{abstract}
Let $w(\theta)$ be a positive weight function on the interval $[-\pi, \pi]$ and associate the positive definite inner product on the unit circle of the complex plane by $\langle F, G\rangle_{w}=$ $\frac{1}{2 \pi} \int_{-\pi}^{\pi} F\left(e^{i \theta}\right) \overline{G\left(e^{i \theta}\right)} w(\theta) \mathrm{d} \theta$. For a sequence of points $\left\{\alpha_{k}\right\}_{k=1}^{\infty}$ included in a compact subset of the open unit disk, we consider the orthogonal rational functions (ORF) $\left\{\phi_{k}\right\}_{k=0}^{\infty}$ that are obtained by orthogonalization of the sequence $\left\{1, z / \pi_{1}, z^{2} / \pi_{2}, \ldots\right\}$ where $\pi_{k}(z)=\prod_{j=1}^{k}\left(1-\bar{\alpha}_{j} z\right)$, with respect to this inner product.

In this paper we prove that $\mathbf{s}_{n}(z)-\mathbf{S}_{n}(z)$ tends to zero in $|z| \leq 1$ if $n$ tends to $\infty$, where $\mathbf{s}_{n}$ is the $n$th partial sum of the expansion of a bounded analytic function $F$ in terms of the ORF $\left\{\phi_{k}\right\}_{k=0}^{\infty}$ and $\mathbf{S}_{n}$ is the $n$th partial sum of the ordinary power series expansion of $F$. The main condition on the weight is that it satisfies a Dini-Lipschitz condition and that it is bounded away from zero. This generalizes a theorem given by Szego" in the polynomial case, that is when all $\alpha_{k}=0$.

As an important consequence we find that under the above conditions on the weight $w$ and the points $\left\{\alpha_{k}\right\}_{k=1}^{\infty}$, the Cesàro means of the series $\mathbf{s}_{n}$ converge uniformly to the function $F$ in $|z| \leq 1$ if the boundary function $f(\theta):=F\left(e^{i \theta}\right)$ is continous on $[0,2 \pi]$. This can be seen as a generalization of Fejér's Theorem.
\end{abstract}

Key words: equiconvergence, Fejér theorem, orthogonal rational functions AMS Subject classification: 42A20, 26A15

\footnotetext{
1 This work is partially supported by the Fund for Scientific Research (FWO), project "Orthogonal systems and their applications", grant \#G.0278.97 and the Belgian Programme on Interuniversity Poles of Attraction, initiated by the Belgian State, Prime Minister's Office for Science, Technology and Culture. The scientific responsibility rests with the authors.

2 Email: $\{$ Patrick.Vangucht |Adhemar.Bultheel\}@cs.kuleuven.ac.be
} 


\section{Introduction}

First we define the spaces of rational functions that play a central role in this paper. For a given sequence of points $\left\{\alpha_{k}\right\}_{k=1}^{\infty}$, we define the factors

$$
\pi_{0}=1, \quad \pi_{n}=\prod_{i=1}^{n}\left(1-\bar{\alpha}_{i} z\right), \quad n=1,2, \ldots
$$

If $\Pi_{n}$ denotes the space of all polynomials of degree at most $n$, then we set

$$
\mathcal{L}_{n}=\left\{p_{n}(z) / \pi_{n}(z): p_{n} \in \Pi_{n}\right\}
$$

There are several ways to give bases for these spaces. We shall use here the Blaschke products defined as

$$
B_{0}=1, \quad B_{n}(z)=\zeta_{1}(z) \cdots \zeta_{n}(z), \quad n=1,2, \ldots
$$

with Blaschke factors, defined for $k=1,2, \ldots$ as

$$
\zeta_{k}(z)=z_{k} \frac{z-\alpha_{k}}{1-\bar{\alpha}_{k} z}, \quad z_{k}= \begin{cases}\bar{\alpha}_{k} /\left|\alpha_{k}\right|, & \text { if } \alpha_{k} \neq 0 \\ 1, & \text { otherwise }\end{cases}
$$

Then it is clear that $\mathcal{L}_{n}=\operatorname{span}\left\{B_{0}, \ldots, B_{n}\right\}$.

We shall now consider some weight function $w$ on $[-\pi, \pi]$ and the corresponding inner product

$$
\langle F, G\rangle_{w}=\frac{1}{2 \pi} \int_{-\pi}^{\pi} F\left(e^{i \theta}\right) \overline{G\left(e^{i \theta}\right)} w(\theta) \mathrm{d} \theta
$$

and orthogonalize the basis $B_{0}, B_{1}, \ldots$ with respect to this inner product, to get the system of orthonormal rational functions (ORF) $\phi_{0}, \phi_{1}, \ldots$ Thus

$$
\left\langle\phi_{k}, \phi_{l}\right\rangle_{w}=\delta_{k l}
$$

where $\delta_{k l}$ is the Kronecker-delta.

We suppose that the weight function $w$ we consider here satisfies a DiniLipschitz condition

$$
|w(\theta+\delta)-w(\theta)|<L|\log \delta|^{-1-\lambda}
$$


where $L$ is a fixed positive constant and $\lambda>0$. If we also assume that $w$ is non-zero, we can find positive numbers $m$ and $M$ such that

$$
0<m \leq w(\theta) \leq M<\infty, \quad \forall \theta \in[-\pi, \pi] .
$$

A related condition considered in $[5$, p. 227] is

$$
\int_{0}^{\pi} \frac{\omega(w ; \delta)}{\delta} \mathrm{d} \delta<\infty
$$

where $\omega(w ; \delta)$ denotes the modulus of continuity

$$
\omega(w ; \delta)=\sup \{|w(x)-w(y)|:|x-y|<\delta\} .
$$

Note that the form (1.2) of the Dini-Lipschitz condition is stronger than (1.4) in the sense that (1.2) implies (1.4).

A third form of the Dini-Lipschitz condition can be found in [4]

$$
\lim _{\delta \rightarrow 0} \omega(w ; \delta) \log (\delta)=0 .
$$

This one is weaker than (1.4) in the sense that (1.4) implies (1.5). So we have the following implications

$$
(1.2) \Rightarrow(1.4) \Rightarrow(1.5)
$$

Unless stated otherwise, we mean the condition (1.2) when we refer to the Dini-Lipschitz condition. It is the most informative since the parameter $\lambda$ describes the speed of convergence precisely. Note that in the literature it is usually (1.5) that is referred to as the Dini-Lipschitz condition.

We denote the unit circle as $\mathbb{T}=\{z \in \mathbb{C}:|z|=1\}$ and the open unit disk as $\mathbb{D}=\{z \in \mathbb{C}:|z|<1\}$. The closure is denoted as $\overline{\mathbb{D}}=\mathbb{D} \cup \mathbb{T}$.

Because $\log w \in L^{1}(\mathbb{T})$, we can define the spectral factor

$$
\sigma(z)=\exp \left\{\frac{1}{4 \pi} \int_{-\pi}^{\pi} \frac{z+\xi}{z-\xi} \log w(\theta) \mathrm{d} \theta\right\}, \quad \xi=e^{i \theta} .
$$

This $\sigma$ is an outer function in $H^{2}(\mathbb{D})$ which has a nontangential boundary value on $\mathbb{T}$. Also $\left|\sigma\left(e^{i \theta}\right)\right|^{2}=w(\theta)$ a.e. 
Without loss of generality, we shall also assume that $\int_{-\pi}^{\pi} w(\theta) \mathrm{d} \theta=2 \pi$, so that we have $\phi_{0}=1$. For the sequence of points $\left\{\alpha_{k}\right\}_{k=1}^{\infty}$ we make the following two assumptions:

(1) The $\left\{\alpha_{k}\right\}_{k=1}^{\infty}$ are compactly included in the open unit disk, thus $\left|\alpha_{k}\right| \leq$ $1-d$ with $d$ a positive number not depending on $k$;

(2) The counting measures $\nu_{n}^{\alpha}$, that is the discrete measure $\nu_{n}^{\alpha}:=\frac{1}{n} \sum_{i=1}^{n} \delta_{\alpha_{i}}$ that assigns a mass $1 / n$ to the points $\alpha_{k}$ for $k=1, \ldots, n$, has a weak star limit $\nu^{\alpha}$, that is $\lim _{n \rightarrow \infty} \int F(z) \mathrm{d} \nu_{n}^{\alpha}(z)=\int F(z) \mathrm{d} \nu^{\alpha}(z)$ for all continuous functions $F$. This is a mild condition on the distribution of the $\left\{\alpha_{k}\right\}_{k=1}^{\infty}$.

Under these conditions on the weight $w$ and on the points $\left\{\alpha_{k}\right\}_{k=1}^{\infty}$, we gave in a previous paper [3], the asymptotics for the ORF $\left\{\phi_{k}\right\}_{k=0}^{\infty}$ on the unit circle. We even got the rate of convergence, namely $O(\log n)^{-\lambda}$. The main result was the following.

Theorem 1.1 Suppose that the weight is uniformly bounded and satisfies a Dini-Lipschitz condition (1.2). Furthermore, suppose that the above conditions on the $\left\{\alpha_{k}\right\}_{k=1}^{\infty}$ are satisfied. Then, as $n \rightarrow \infty$, we have uniformly for $z \in \mathbb{D} \cup \mathbb{T}$

$$
\rho_{n} \frac{\left(1-\bar{\alpha}_{n}\right) \phi_{n}^{*}(z)}{\sqrt{1-\left|\alpha_{n}\right|^{2}}}=\frac{1}{\sigma(z)}+O(\log n)^{-\lambda}
$$

where $\phi_{n}^{*}(z)=B_{n}(z) \overline{\phi_{n}(1 / \bar{z})}$ denotes the generalized reciprocal of the $\boldsymbol{O R} \boldsymbol{F}$ $\phi_{n}$, and $\rho_{n} \in \mathbb{T}$ is a normalizing constant.

We shall in this paper consider the uniform convergence of a general Fourier expansion with respect to the $\mathbf{O R F}\left\{\phi_{k}\right\}_{k=0}^{\infty}$. Consider a function $F(z)$ analytic in $|z|<1$ that has a (bounded) non-tangential boundary value for $|z| \rightarrow 1$, which we also denote as $F(z)$. Obviously, since we have an orthonormal system, we get for such a function the formal expansion $\sum_{k=0}^{\infty}\left\langle F, \phi_{k}\right\rangle_{w} \phi_{k}$. Let us denote the partial sums as

$$
\mathbf{s}_{n}(z)=\sum_{k=0}^{n}\left\langle F, \phi_{k}\right\rangle_{w} \phi_{k}(z)
$$

We are interested in finding conditions under which $\mathbf{s}_{n}$ converges uniformly to $F$ in $\overline{\mathbb{D}}$. It is clear that in the simplest possible case, that is when we consider the weight $w \equiv 1$ and set all $\alpha_{k}=0$, then the $\mathbf{O R F}$ are just the powers $f_{k}(z)=z^{k}$, and even then the uniform convergence of $\mathbf{s}_{n}$ to $F(z)$ is not guaranteed on $\mathbb{T}$. Indeed, in this case we obtain the Maclaurin series expansion of $F$. Thus if $F$ is analytic in the open unit disk, then we have uniform convergence there, but it is not guaranteed that there is uniform convergence on the circle itself. Let us introduce some notation for this special 
case. The inner product with $w \equiv 1$, i.e., with the Lebesgue measure, is denoted as $\langle\cdot, \cdot\rangle$, rather than $\langle\cdot, \cdot\rangle_{1}$. The partial sum of the expansion with respect to the orthogonal functions $f_{k}(z) \equiv z^{k}$ is denoted as

$$
\mathbf{S}_{n}(z)=\sum_{k=0}^{n}\left\langle F, f_{k}\right\rangle f_{k}(z)
$$

Rather than proving that $\mathbf{s}_{n}$ converges to $F$, we shall first prove that $\mathbf{s}_{n}-\mathbf{S}_{n}$ converges uniformly to zero under a somewhat stronger Dini-Lipschitz condition on the weight $w$ : it shall be assumed that $\lambda>1$. For the function $F$ it will only be required that it is regular and bounded in $\mathbb{D}$. This means that $F \in H^{\infty}(\mathbb{D})$.

The main objective of this paper is to prove the following theorem.

Theorem 1.2 Let $w$ be a non-zero weight function on $[-\pi, \pi]$ that satisfies the following Dini-Lipschitz condition $(\delta>0)$

$$
|w(\theta+\delta)-w(\theta)|<L|\log \delta|^{-1-\lambda}
$$

where $L>0$ and $\lambda>1$ are fixed numbers. Thus (1.3) holds. For the $\left\{\alpha_{k}\right\}_{k=1}^{\infty}$ we assume that they are all in a compact subset of $\mathbb{D}$ and that the associated counting measure $\nu_{n}^{\alpha}$ converges in the weak star topology to the measure $\nu^{\alpha}$.

Let $F \in H^{\infty}(\mathbb{D})$. If $\mathbf{s}_{n}$ denotes the $n$th partial sum of the expansion of the boundary function $F(z), z \in \mathbb{T}$, in terms of the $\boldsymbol{O R} \boldsymbol{F}\left\{\phi_{k}\right\}_{k=0}^{\infty}$ associated with $w$, and if $\mathbf{S}_{n}$ is the nth partial sum of the ordinary power series expansion of $F$, then we have

$$
\lim _{n \rightarrow \infty}\left\{\mathbf{s}_{n}(z)-\mathbf{S}_{n}(z)\right\}=0
$$

uniformly in the whole closed unit disc $\overline{\mathbb{D}}$.

In the second section we deduce the lemmas we need to prove this theorem. The proof is then given in the third section. In the last section a number of corollaries of our main theorem are given. The most important result here is a generalization of Fejér's Theorem [4] stating that under the conditions of Theorem 1.2 for the weight $w$ and the points $\left\{\alpha_{k}\right\}_{k=1}^{\infty}$, the Cesàro means of the partial sums $\mathbf{s}_{n}$ converge uniformly on $\mathbb{D} \cup \mathbb{T}$ to the function $F$ if this function $F$ is bounded and analytic in $\mathbb{D}$ and if its boundary function $f(\theta):=F\left(e^{i \theta}\right)$ is continous on $[0,2 \pi]$. This means that $F$ is in the disc algebra $\mathcal{A}(\mathbb{D})$.

Before entering the next section, let us recall that throughout this paper, the $\left\{\alpha_{k}\right\}_{k=1}^{\infty}$ and the ORF $\left\{\phi_{k}\right\}_{k=0}^{\infty}$ will always satisfy the properties given 
above. Furthermore, we define for any complex function $F$ the para-hermitian conjugate as $F_{*}(z)=\overline{F(1 / \bar{z})}$ and we define $\mathcal{L}_{n *}=\left\{F: F_{*} \in \mathcal{L}_{n}\right\}$. Finally we define for a function $F_{n} \in \mathcal{L}_{n} \backslash \mathcal{L}_{n-1}$ the generalized reciprocal function $F^{*}$ as $F_{n}^{*}(z)=B_{n}(z) F_{n *}(z)$.

\section{Some preliminaries}

Notice that it is sufficient to discuss the statement for $z \in \mathbb{T}$, because we are dealing with analytic functions. The following integral expressions for the partial sums are easy to obtain. Setting $\xi=e^{i \theta}$ we get

$$
\begin{aligned}
\mathbf{s}_{n}(z) & =\sum_{i=0}^{n}\left\langle F, \phi_{i}\right\rangle_{w} \phi_{i}(z)=\sum_{i=0}^{n} \phi_{i}(z) \frac{1}{2 \pi} \int_{-\pi}^{\pi} F(\xi) \overline{\phi_{i}(\xi)} w(\theta) \mathrm{d} \theta \\
& =\frac{1}{2 \pi} \int_{-\pi}^{\pi} F(\xi) k_{n}(w ; z, \xi) w(\theta) \mathrm{d} \theta .
\end{aligned}
$$

We have used the notation $k_{n}(w ; z, \xi)$ to denote the kernel $k_{n}(w ; z, \xi):=$ $\sum_{i=0}^{n} \phi_{i}(x) \overline{\phi_{i}(\xi)}$. Note that $k_{n}$ is the reproducing kernel for $\mathcal{L}_{n}$ with respect to the inner product $\langle\cdot, \cdot\rangle_{w}$. For the power series expansion we have (recall $\left.f_{k}(z)=z^{k}\right)$

$$
\begin{aligned}
\mathbf{S}_{n}(z) & =\sum_{i=0}^{n}\left\langle F, f_{i}\right\rangle z^{i}=\sum_{i=0}^{n} z^{i} \frac{1}{2 \pi} \int_{-\pi}^{\pi} F(\xi) \bar{\xi}^{i} \mathrm{~d} \theta \\
& =\frac{1}{2 \pi} \int_{-\pi}^{\pi} F(\xi) \frac{1-(\bar{\xi} z)^{n+1}}{1-\bar{\xi} z} \mathrm{~d} \theta .
\end{aligned}
$$

Here the reproducing kernel is

$$
K_{n}(z, \xi)=\sum_{i=0}^{n} f_{i}(z) \overline{f_{i}(\xi)}=\sum_{i=0}^{n} z^{i} \bar{\xi}^{i}=\frac{1-(\bar{\xi} z)^{n+1}}{1-\bar{\xi} z}
$$

We denote with $\Delta_{n}(z, \xi)$ the difference of the kernels multiplied by their respective weight functions

$$
\Delta_{n}(z, \xi)=k_{n}(w ; z, \xi) w(\theta)-\frac{1-(\bar{\xi} z)^{n+1}}{1-\bar{\xi} z} .
$$

Thus our main theorem will be proved if we show that (recall $\xi=e^{i \theta}$ )

$$
\lim _{n \rightarrow \infty} \int_{-\pi}^{\pi} F(\xi) \Delta_{n}(z, \xi) \mathrm{d} \theta=0, \quad \forall z \in \mathbb{T} .
$$


We now give a sequence of lemma's that are necessary to prove the main theorem.

From [2] we get the Christoffel-Darboux relations for orthonormal rational functions.

Lemma 2.1 The following relation holds between the reproducing kernel $k_{n}(w ; z, \xi)$ and the $\boldsymbol{O R F}\left\{\phi_{k}\right\}_{k=0}^{\infty}$ :

$$
k_{n}(w ; z, \xi)=\frac{\phi_{n+1}^{*}(z) \overline{\phi_{n+1}^{*}(\xi)}-\phi_{n+1}(z) \overline{\phi_{n+1}(\xi)}}{1-\zeta_{n+1}(z) \overline{\zeta_{n+1}(\xi)}} .
$$

Remember that the $\zeta_{n}$ are the Blaschke factors (1.1).

We recall the simple fact that if $w$ satisfies a Dini-Lipschitz condition then also $1 / w$ satisfies a Dini-Lipschitz condition (see $[3,7]$ ).

We now prove the following lemma, which says that also the spectral factor $\sigma$ satisfies a Dini-Lipschitz condition.

Lemma 2.2 If the weight $w$ satisfies the Dini-Lipschitz condition (1.6), then the spectral factor $\sigma$ satisfies the following Dini-Lipschitz condition

$$
|s(\theta+\delta)-s(\theta)|<L^{\prime}|\log \delta|^{-\lambda}
$$

where $s(\theta):=\sigma\left(e^{i \theta}\right)$ and where $L^{\prime}$ is a positive constant and $\lambda>1$ is the same as in (1.6).

PROOF. This lemma was proved in [7]. It relies on a Jackson [6] Theorem for trigonometric polynomials, usually referred to as the Jackson III Theorem [4, p 144]. We give a brief sketch of the proof.

Let $n$ be an arbitrary positive integer. Applying the Jackson Theorem to the function $1 / w$, we find a trigonometric polynomial $g_{n}$ of order $n$, so that

$$
\left|w(\theta)-1 / g_{n}(\theta)\right|<P(\log n)^{-1-\lambda},
$$

where we used the Dini-Lipschitz condition of the function $1 / w$. We know that there exists a polynomial $H_{n}$ of degree $n$, so that $g_{n}(\theta)=\left|H_{n}\left(e^{i \theta}\right)\right|^{2}$. We can show that $[7]$

$$
\left|\sigma(z)-1 / H_{n}(z)\right|<Q(\log n)^{-\lambda},
$$


uniformly for $|z| \leq 1$. The constant $Q$ only depends on the minimum and maximum of the weight $w$ as well as on $L$ and $\lambda$, the parameters of the DiniLipschitz condition (1.6). Making use of (2.1) we obtain

$$
\left|\sigma\left(e^{i(\theta+\delta)}\right)-\sigma\left(e^{i \theta}\right)\right|<2 Q(\log n)^{-\lambda}+\left|H_{n}\left(e^{i(\theta+\delta)}\right)^{-1}-H_{n}\left(e^{i \theta}\right)^{-1}\right| .
$$

By the Theorem of Bernstein [7, Theorem 1.22.1] the second term on the right-hand side is equal to $\delta O(n)$. So we found the bound $O(\log n)^{-\lambda}+\delta O(n)$. When we put $n=O\left(\delta^{-1}|\log \delta|^{-\lambda}\right)$ the statement of the lemma follows.

The previous proof can be given using rational functions as well. Indeed, a Jackson III type Theorem was derived in [1, Lemma 4.6] and except for technicalities, the proof can be given along the same line. This is however an unnecessary complication.

We remark here that by (2.1) and using the same kind of arguments as above, we can obtain the following more general inequality

$$
\left|\sigma\left(z_{1}\right)-\sigma\left(z_{2}\right)\right|<L^{\prime}|\log | z_{1}-\left.z_{2}\right|^{-\lambda}, \quad \forall z_{1}, z_{2} \in \mathbb{D} \cup \mathbb{T} .
$$

We now derive an approximation of the $\mathbf{O R F}\left\{\phi_{k}\right\}_{k=0}^{\infty}$ in terms of the spectral factor $\sigma$.

Lemma 2.3 With the notations of Theorem 1.1, we find for $z \in \mathbb{D} \cup \mathbb{T}$ and for $n \rightarrow \infty$

$$
\begin{aligned}
& \phi_{n}^{*}(z)=\bar{\rho}_{n} \frac{\sqrt{1-\left|\alpha_{n}\right|^{2}}}{1-\bar{\alpha}_{n} z} \frac{1}{\sigma(z)}+O(\log n)^{-\lambda} ; \\
& \phi_{n}(z)=\rho_{n} z_{n} \frac{\sqrt{1-\left|\alpha_{n}\right|^{2}}}{1-\bar{\alpha}_{n} z} \frac{z B_{n-1}(z)}{\sigma_{*}(z)}+O(\log n)^{-\lambda},
\end{aligned}
$$

where $\rho_{n} \in \mathbb{T}$ is for normalization and $z_{n} \in \mathbb{T}$ as defined in (1.1).

PROOF. The first relation is simply a rewriting of the result from Theorem 1.1. The second equation can be obtained by the definition of the generalized reciprocal of the function.

$$
\begin{aligned}
\phi_{n}(z) & =B_{n}(z)\left[\phi_{n}^{*}(z)\right]_{*} \\
& =B_{n}(z) \rho_{n} \frac{\sqrt{1-\left|\alpha_{n}\right|^{2}}}{1-\alpha_{n} / z} \frac{1}{\sigma_{*}(z)}+O(\log n)^{-\lambda}
\end{aligned}
$$




$$
=\rho_{n} z_{n} \frac{\sqrt{1-\left|\alpha_{n}\right|^{2}}}{1-\bar{\alpha}_{n} z} \frac{z B_{n-1}(z)}{\sigma_{*}(z)}+O(\log n)^{-\lambda} .
$$

This proves the lemma.

The next lemma gives a boundary for the modulus of the kernel on $\mathbb{T}$.

Lemma 2.4 If $k_{n}(w ; z, \zeta)$ denotes the kernel for the $\boldsymbol{O R} \boldsymbol{F}\left\{\phi_{k}\right\}_{k=0}^{\infty}$, then for $n \rightarrow \infty$

$$
\left|k_{n}(w ; z, \zeta)\right| \leq O(n), \quad z, \zeta \in \mathbb{D} \cup \mathbb{T}
$$

PROOF. From [3, Lemma 4.8] we find (for $k \rightarrow \infty$ )

$$
\max _{t \in \mathbb{T}}\left|\phi_{k}(t)\right|=O(1) .
$$

Because $\phi_{k}$ is analytic in $\mathbb{D}$, we find for $z, \zeta \in \mathbb{D} \cup \mathbb{T}$

$$
\left|k_{n}(w ; z, \zeta)\right| \leq \sum_{k=0}^{n}\left|\phi_{k}(z)\right|\left|\overline{\phi_{k}(\zeta)}\right| \leq \sum_{k=0}^{n} O(1)=O(n) .
$$

For a more detailed expression of this bound see [3, Lemma 4.6].

An asymptotic expression for the kernel can be given as in the next lemma. Its explicit form is not really needed in the sequel, but it gives an introduction to the proof of Lemma 2.6 and the result is interesting enough in its own right.

Lemma 2.5 Let $k_{n}(w ; z, \xi)$ be the kernel for the $\boldsymbol{O R} \boldsymbol{F}\left\{\phi_{k}\right\}_{k=0}^{\infty}$. Let the assumptions made above on the weight $w$ and the points $\alpha$ be satisfied. Then for $z \in \overline{\mathbb{D}}=\mathbb{D} \cup \mathbb{T}$, we have uniformly for $\xi \in \overline{\mathbb{D}} \backslash\{z\}$ as $n \rightarrow \infty$

$$
k_{n}(w ; z, \xi)=\frac{1}{1-z \bar{\xi}}\left[\frac{1}{\sigma(z) \overline{\sigma(\xi)}}-\frac{z \bar{\xi} B_{n}(z) \overline{B_{n}(\xi)}}{\sigma_{*}(z) \overline{\sigma_{*}(\xi)}}\right]+\frac{O(\log n)^{-\lambda}}{1-z \bar{\xi}} .
$$

PROOF. This follows immediately from substituting the asymptotics for the ORF of Lemma 2.3 into the Christoffel-Darboux relation of Lemma 2.1 and by using the relation

$$
1-\zeta_{n+1}(z) \overline{\zeta_{n+1}(\xi)}=\frac{(\xi-z)\left(1-\left|\alpha_{n+1}\right|^{2}\right)}{\left(1-\bar{\alpha}_{n+1} z\right)\left(\xi-\alpha_{n+1}\right)}
$$

for the denominator of the Chistoffel-Darboux relation. 
The next lemma gives us an explicit form of $\Delta_{n}(z, \xi)$ for $z, \xi \in \mathbb{T}$.

Lemma 2.6 For $z \in \mathbb{T}$ the following equality holds uniformly for $\xi$ in compact subsets of $\mathbb{T} \backslash\{z\}$ and $n \rightarrow \infty$

$$
\Delta_{n}(z, \xi)=\frac{\sigma(\xi) / \sigma(z)-1}{1-\bar{\xi} z}-(\bar{\xi} z)^{n+1} \frac{\frac{\overline{\sigma(\xi)}}{\frac{\sigma(z)}{\sigma(z)}} \frac{B_{n}(z)}{z^{n}} \frac{\xi^{n}}{B_{n}(\xi)}-1}{1-\bar{\xi} z}+\frac{O(\log n)^{-\lambda}}{1-\bar{\xi} z} .
$$

PROOF. Suppose $z, \xi \in \mathbb{T}$, but $z \neq \xi=e^{i \theta}$. From the first ChristoffelDarboux relation (Lemma 2.1) we find

$$
\Delta_{n}(z, \xi)=\frac{\phi_{n+1}^{*}(z) \overline{\phi_{n+1}^{*}(\xi)}-\phi_{n+1}(z) \overline{\phi_{n+1}(\xi)}}{1-\zeta_{n+1}(z) \overline{\zeta_{n+1}(\xi)}}|\sigma(\xi)|^{2}-\frac{1-(\bar{\xi} z)^{n+1}}{1-\bar{\xi} z}
$$

For the first term, we use the asymptotic expression that was obtained in the previous lemma, applying it for $z, \xi \in \mathbb{T}$ so that $\sigma_{*}(z)=\overline{\sigma(z)}$ and $\sigma_{*}(\xi)=\overline{\sigma(\xi)}$. This gives

$$
\begin{aligned}
& \Delta_{n}(z, \xi)=\frac{\sigma(\xi) / \sigma(z)-\frac{\overline{\sigma(\xi)}}{\sigma(z)} \frac{z B_{n}(z)}{\xi B_{n}(\xi)}}{1-\bar{\xi} z}-\frac{1-(\bar{\xi} z)^{n+1}}{1-\bar{\xi} z}+\frac{O(\log n)^{-\lambda}}{1-\bar{\xi} z} \\
&=\frac{\sigma(\xi) / \sigma(z)-1}{1-\bar{\xi} z}-(\bar{\xi} z)^{n+1} \\
& \frac{\frac{\overline{\sigma(\xi)}}{\overline{\sigma(z)}} \frac{B_{n}(z)}{z^{n}} \frac{\xi^{n}}{B_{n}(\xi)}-1}{1-\bar{\xi} z}+\frac{O(\log n)^{-\lambda}}{1-\bar{\xi} z} .
\end{aligned}
$$

This proves the lemma.

\section{Proof of the main theorem}

Now we are able to prove the main theorem

Proof of Theorem 1.2 We have to prove that for $F \in H^{\infty}(\mathbb{D})$

$$
\lim _{n \rightarrow \infty} \int_{-\pi}^{\pi} F(\xi) \Delta_{n}(z, \xi) \mathrm{d} \theta=0, \quad \xi=e^{i \theta}
$$

uniformly for $z \in \mathbb{T}$. We split the integral into two parts

$$
\int_{E} F(\xi) \Delta_{n}(z, \xi) \mathrm{d} \theta+\int_{E^{\prime}} F(\xi) \Delta_{n}(z, \xi) \mathrm{d} \theta
$$


where $E=E(n, \varepsilon, z)$ is the set $\left\{\xi \in \mathbb{T}:|z-\xi| \geq \varepsilon n^{-1}\right\}$ and $E^{\prime}=E^{\prime}(n, \varepsilon, z)$ is the complementary set $\mathbb{T} \backslash E$. Here $\varepsilon$ is an arbitrary small positive number.

The second integral in (3.1) is easy to bound. By Lemma 2.4 we find that $\Delta_{n}(z, \xi)=O(n)$. So we have

$$
\int_{E^{\prime}} F(\xi) \Delta_{n}(z, \xi) \mathrm{d} \theta=O(n) \varepsilon n^{-1}=\varepsilon O(1)
$$

This is arbitrarily small as $\varepsilon \rightarrow 0$.

Before looking at the first integral, we take a look at the integrals $\left(\xi=e^{i \theta}\right)$

$$
\int_{-\pi}^{\pi} F(\xi) \frac{\sigma(\xi) / \sigma(z)-1}{1-\bar{\xi} z} \mathrm{~d} \theta \quad \text { and } \quad \int_{-\pi}^{\pi} F(\xi)(\bar{\xi} z)^{n+1} \frac{\frac{\overline{\sigma(\xi)}}{\overline{\sigma(z)}} \frac{B_{n}(z)}{z^{n}} \frac{\xi^{n}}{B_{n}(\xi)}-1}{1-\bar{\xi} z} \mathrm{~d} \theta
$$

Because $F \in H^{\infty}(\mathbb{D})$ and because also the rest of the integrand is analytic inside the unit disk (the pole $\xi=z$ is canceled by a zero in the numerator) we can apply Cauchy's Theorem to find the following result

$$
\frac{1}{2 \pi} \int_{-\pi}^{\pi} F(\xi) \frac{\sigma(\xi) / \sigma(z)-1}{1-\bar{\xi} z} \mathrm{~d} \theta=\frac{1}{2 \pi i} \int_{|\xi|=1} F(\xi) \frac{\sigma(\xi) / \sigma(z)-1}{1-\bar{\xi} z} \frac{\mathrm{d} \xi}{\xi}=0
$$

Next we take a look at the function $F_{n}$, defined as $(z \in \mathbb{T}$ is a parameter $)$

$$
F_{n}(\xi):=\frac{\overline{\frac{\sigma(\xi)}{\sigma(z)}} \frac{B_{n}(z)}{z^{n}} \frac{\xi^{n}}{B_{n}(\xi)}-1}{1-\bar{\xi} z} .
$$

We see that the numerator of $F_{n}$ has a simple zero at $\xi=z$. This cancels the simple zero of the denominator and thus $F_{n}$ is a rational function which has all its poles strictly inside the unit disc (this is a consequence of the assumption that the $\left\{\alpha_{k}\right\}_{k=1}^{\infty}$ are all in a compact subset of $\mathbb{D}$ and because $\sigma$ is uniformly bounded). Therefore we can use Cauchy's Theorem to find $\left(\xi=e^{i \theta}\right)$

$$
\int_{-\pi}^{\pi} F_{n}(\xi) \mathrm{d} \theta=0
$$

Thus $F_{n} \in L^{1}(\mathbb{T})$. Because $F$ is bounded, we see that the product $F F_{n}$ is also in $L^{1}(\mathbb{T})$. According to the Riemann-Lebesgue Lemma (see [8, p. 45]), we now 
find

$$
\lim _{n \rightarrow \infty} \int_{-\pi}^{\pi} F(\xi)(\bar{\xi} z)^{n+1} F_{n}(\xi) \mathrm{d} \theta=0
$$

When we combine Lemma 2.6, (3.2) and (3.3), we find

$$
\int_{-\pi}^{\pi} F(\xi)\left(\Delta_{n}(z, \xi)-\frac{O(\log n)^{-\lambda}}{1-\bar{\xi} z}\right) \mathrm{d} \theta=o(1) .
$$

We use this equation to bound the first integral in (3.1) as follows

$$
\begin{aligned}
& \int_{E} F(\xi) \Delta_{n}(z, \xi) \mathrm{d} \theta=O(\log n)^{-\lambda} \int_{E} \frac{\mathrm{d} \theta}{|1-\bar{\xi} z|} \\
&-\int_{E^{\prime}} F(\xi)\left\{\frac{\sigma(\xi) / \sigma(z)-1}{1-\bar{\xi} z}-(\bar{\xi} z)^{n+1} \frac{\overline{\frac{\sigma(\xi)}{\sigma(z)}} \frac{B_{n}(z)}{z^{n}} \frac{\xi^{n}}{B_{n}(\xi)}-1}{1-\bar{\xi} z}\right\} \mathrm{d} \theta+o(1) \\
&=O(\log n)^{1-\lambda}-O(1) \int_{E^{\prime}} \frac{|\log | z-\left.\xi\right|^{-\lambda}}{1-\bar{\xi}_{z}} \mathrm{~d} \theta \\
& \quad+\int_{E^{\prime}} F(\xi)(\bar{\xi} z)^{n+1} F_{n}(\xi) \mathrm{d} \theta+o(1) \\
&=O(\log n)^{1-\lambda}+o(1)=o(1), \quad n \rightarrow \infty .
\end{aligned}
$$

The second equality follows from (2.2) and the third from (3.3). This proves our statement.

\section{Some important consequences}

In this last section we give some consequences of Theorem 1.2. We have shown that certain some conditions on the weight $w$ and the points $\left\{\alpha_{k}\right\}_{k=1}^{\infty}$ and under some mild conditions for the function $F$, the general ORF-Fourier series and the ordinary Fourier series for $F$ behave in the same way. Thus, if we impose extra conditions on the function $F$ that guarantee that $\mathbf{S}_{n}$ converges uniformly to $F$ in $\overline{\mathbb{D}}=\mathbb{D} \cup \mathbb{T}$, then also $\mathbf{s}_{n}$ shall convergerge uniformly to $F$ in $\overline{\mathbb{D}}$.

Two examples are given below: either the boundary function $f(\theta):=F\left(e^{i \theta}\right)$ is $2 \pi$-periodic and satisfies a Dini-Lipschitz condition of the form (1.5) or it is continuous and of bounded variation.

As we mentioned before, since $F$ is analytic in $\mathbb{D}$, it is sufficient to consider convergence on $\mathbb{T}$, since this immediately implies convergence in $\overline{\mathbb{D}}$. Indeed, 
$F-\mathbf{S}_{n}$ is analytic in $\mathbb{D}$ and by the maximum modulus Theorem, the maximum is reached on $\mathbb{T}$.

Since we are interested in uniform convergence of the generalized Fourier series $\mathbf{s}_{n}$ on the unit circle $\mathbb{T}$, we need to impose some constraints on the boundary function $f(\theta):=F\left(e^{i \theta}\right)$.

If we assume Dini-Lipschitz conditions for the boundary function $f$ we get the following generalization of the Dini-Lipschitz Theorem [4, p 146].

Theorem 4.1 (Dini-Lipschitz) Suppose that the weight $w$ and the points $\left\{\alpha_{k}\right\}_{k=1}^{\infty}$ satisfy the conditions of theorem 1.2. If $F \in \mathcal{A}(\mathbb{D})$ and its boundary function $f(\theta):=F\left(e^{i \theta}\right)$ satisfies a Dini-Lipschitz condition of the form (1.5)

$$
\omega(f ; \delta) \log (\delta) \rightarrow 0, \quad \delta \rightarrow 0
$$

then the series $\mathbf{s}_{n}$ converges uniformly to $F$ in $\overline{\mathbb{D}}$.

PROOF. This can be proved by combining Theorem 1.2 and the Dini-Lipschitz Theorem for ordinary Fourier series (see e.g. [4, p 146] or [8, p 63]). This theorem states that under the same conditions the series $\mathbf{S}_{n}$ converges uniformly to $F$ on $\mathbb{T}$.

When the boundary function $f$ is of bounded variation we can find the following.

Theorem 4.2 Suppose that the weight $w$ and the points $\left\{\alpha_{k}\right\}_{k=0}^{\infty}$ satisfy the conditions of Theorem 1.2. If $F \in \mathcal{A}(\mathbb{D})$ and its boundary function $f(\theta):=$ $F\left(e^{i \theta}\right)$ is of bounded variation over $[0,2 \pi]$, then the series $\mathbf{s}_{n}$ converges uniformly to $F$ in $\overline{\mathbb{D}}$.

PROOF. This can also be proved by combining two theorems, namely Theorem 1.2 and the Dirichlet-Jordan-test [8, Theorem 8.14] that states that under the same conditions the series $\mathbf{S}_{n}$ converges uniformly to $F$ on $\mathbb{T}$.

Finally we generalize the Fejér Theorem (see e.g. [8, p 89] or [4, p 123]) which says that the Cesàro means of the Fourier series $\mathbf{S}_{n}$ converges uniformly for a continuous function. We now prove that under our conditions on the weight $w$ and the points $\left\{\alpha_{k}\right\}_{k=1}^{\infty}$, this also holds in the general case of an ORF-Fourier series.

Theorem 4.3 (Fejér) Suppose that the weight $w$ and the points $\left\{\alpha_{k}\right\}_{k=1}^{\infty}$ 
satisfy the conditions of Theorem 1.2. If $F \in \mathcal{A}(\mathbb{D})$, then we find ( $\sigma_{N}$ denotes the Nth Cesàro mean of $\mathbf{s}_{n}$ )

$$
\lim _{N \rightarrow \infty} \max _{z \in \mathbb{T}}\left|F(z)-\sigma_{N}(z)\right|=0 .
$$

Thus the Cesàro means of $\mathbf{s}_{n}$ converge uniformly to the function $F$ in $\overline{\mathbb{D}}$.

PROOF. This is a combination of Theorem 1.2 and the well-known Fejér Theorem, that states that if $f(\theta)=F\left(e^{i \theta}\right)$ is continous, then the Cesàro means of $\mathbf{S}_{n}$ converge uniformly to $F$ in $\overline{\mathbb{D}}$.

\section{Acknowledgements}

We thank the referee for the suggestion to include the asymptotics of the reproducing kernel given in Lemma 2.5.

\section{References}

[1] A. Bultheel, P. González-Vera, E. Hendriksen, and O. Njåstad. On the convergence of multipoint Padé-type approximants and quadrature formulas associated with the unit circle. Numer. Algorithms, 13:321-344, 1996.

[2] A. Bultheel, P. González-Vera, E. Hendriksen, and O. Njåstad. Orthogonal rational functions, volume 5 of Cambridge Monographs on Applied and Computational Mathematics. Cambridge University Press, 1999.

[3] A. Bultheel and P. Van gucht. Boundary asymptotics for orthogonal rational functions on the unit circle. Acta Applicandae Mathematicae, 1999. Accepted.

[4] E.W. Cheney. Introduction to approximation theory. McGraw Hill, 1966.

[5] G. Freud. Orthogonal polynomials. Pergamon Press, Oxford, 1971.

[6] D. Jackson. The Theory of Approximation, volume 11 of Amer. Math. Soc. Colloq. Publ. Amer. Math. Soc., 1930.

[7] G. Szegö. Orthogonal polynomials, volume 33 of Amer. Math. Soc. Colloq. Publ. Amer. Math. Soc., Providence, Rhode Island, 3rd edition, 1967. First edition 1939.

[8] A. Zygmund. Trigonometric series, vol 1 and 2. Cambridge University Press, Cambridge, 1968. 2nd ed., 1st edition 1935. 